\title{
Sincronização de relógios de pêndulo e metrônomos: um tratamento qualitativo
}

\author{
Synchronization of pendulum clocks and metronomes: a qualitative treatment \\ Ricardo L. Viana*10 \\ ${ }^{1}$ Universidade Federal do Paraná, Departamento de Física, CEP 81531-990, Curitiba, PR, Brasil.
}

Recebido em 03 de Julho de 2020. Aceito em 08 de setembro de 2020.

\begin{abstract}
Os relógios de pêndulo e metrônomos têm em comum o fato de serem osciladores auto-excitados, que exibem oscilações estacionárias, onde um aporte externo de energia compensa as perdas devidas ao amortecimento. No plano de fase, estas oscilações são representadas por trajetórias fechadas chamadas ciclos-limite, e o movimento sobre elas pode ser parametrizado por um ângulo de fase. Neste trabalho investigamos a dinâmica resultante do acoplamento de dois osciladores de fase, usando equações médias para os casos de ressonância. Os resultados dessa análise são utilizados para descrever qualitativamente fenômenos de travamento de fase e sincronização em osciladores de fase, e que explicam resultados observados no acoplamento de relógios de pêndulo e metrônomos. Palavras-chave: sincronização, osciladores de fase, osciladores auto-excitados, ciclos-limite, travamento de fase, relógios de pêndulo, metrônomos.
\end{abstract}

Pendulum clocks and metronomes have in common the fact that they are self-excited oscillators, exhibiting stationary oscillations where an external energy input compensates losses due to damping. In the phase plane these oscillations are represented by closed trajectories called limit cycles, and the motion along them can be parameterized by a phase angle. In this work we investigate the dynamics resulting from the coupling between two phase oscillators, using average equations for the resonant cases. The results from this analysis are used to qualitatively describe phase locking and synchronization phenomena in phase oscillators, explaining results observed in the coupling of pendulum clocks and metronomes.

Keywords: synchronization, phase oscillators, self-excited oscillators, limit cycles, phase locking, pendulum clocks, metronomes.

\section{Introdução}

A sincronização de osciladores acoplados é um fenômeno caracterizado pela equalização das suas fases e/ou frequências, causada pela interação entre os osciladores devido ao seu acoplamento. Como as oscilações, tanto mecânicas como eletromagnéticas e de outros tipos, são onipresentes na Natureza, espera-se que a sincronização seja muito comum em aplicações físicas, biológicas e tecnológicas [1].

Dois exemplos podem ser apresentados à guisa de ilustração. O físico teórico William Strutt (Lord Rayleigh) descreveu um fenômeno de sincronização em sistemas acústicos 2]. Um tubo de órgão produz ondas sonoras na forma de harmônicos cuja frequência depende do seu comprimento. Rayleigh constatou que dois tubos idênticos de órgão, quando colocados lado a lado, podem sincronizar os sons emitidos, podendo até mesmo silenciar mutuamente.

Outro exemplo de sincronização, mas de oscilações eletromagnéticas, está associada à chamada válvula triodo, inventada por volta de 1920 . O gerador do tipo triodo é

*Endereço de correspondência: viana@fisica.ufpr.br um tubo de vácuo no interior do qual é produzida uma corrente elétrica alternada cuja frequência depende dos elementos do circuito ao qual o triodo pertence. Edward Appleton acoplou dois triodos com frequências ligeiramente diferentes, observando a sincronização de suas frequências [3].

Relógios de pêndulo e metrônomos são exemplos importantes de osciladores, pois são relativamente fáceis de encontrar na vida quotidiana (embora cada vez menos comuns devido à revolução digital). A sincronização destes osciladores devido ao seu acoplamento mecânico produz belas ilustrações desse fenômeno. Há vários vídeos na internet com experiências de sincronização de metrônomos apoiados num suporte comum. Além disso, é uma experiência que pode ser feita em sala de aula a médio custo.

Uma descrição matemática abrangente da sincronização destes e de outros sistemas similares é complicada pelas inúmeras situações diferentes que podem aparecer [4. Primeiro temos as diferentes variáveis dinâmicas envolvidas: pressão e deslocamento no caso de tubos de órgão, corrente e tensão elétrica no caso do triodo, ângulo e velocidade angular no caso do relógio de pêndulo, e 
assim por diante; cada qual com diferentes equações de evolução temporal. Em segundo lugar, os mecanismos de acoplamento podem ser bastante complexos, envolvendo propagação de impulsos mecânicos ou eletromagnéticos quase imperceptíveis e de difícil modelagem.

A despeito dessas complexidades, é possível fazer uma descrição qualitativa (ou melhor, semi-quantitativa) que reduz os sistemas aos chamados "osciladores de fase", nos quais o estado do sistema é determinado pelo valor de um ângulo de fase [5]. O acoplamento de dois osciladores de fase pode ser modelado por uma função que embute as complicações do acoplamento.

A caracterização da sincronização entre os osciladores de fase, porém, não depende muito da forma dessa função de acoplamento, e sim do seu comportamento nas condições de ressonância. A descrição resultante é bem mais simples, bastante geral, e esclarece a natureza da sincronização entre os osciladores sem entrar em detalhes técnicos sobre o seu acoplamento [1]. Num trabalho anterior usamos uma descrição similar para abordar a sincronização de um oscilador com um forçamento externo periódico 6 .

O objetivo central deste trabalho é apresentar ao leitor esse tratamento qualitativo da sincronização entre osciladores fracamente acoplados usando osciladores de fase. Os resultados desse tratamento são usados para descrever alguns aspectos da sincronização entre relógios de pêndulo e metrônomos, sem a necessidade de entrar a fundo em modelos matemáticos para os mesmos. Além disso, os resultados podem ser adaptados a uma grande variedade de outros exemplos, incluindo osciladores biológicos.

O presente artigo é estruturado da seguinte forma: na Seção 2 nós descrevemos os relógios de pêndulo e a sua sincronização mútua. Na Seção 3 revisamos alguns aspectos da descrição matemática do pêndulo, enfatizando o papel desempenhado pelo mecanismo de escape do tipo âncora-roda dentada. Na seção 4 abordamos, na mesma linha, a sincronização de metrônomos, e como tratar tanto os relógios de pêndulo como os metrônomos como osciladores de fase. A Seção 5 é dedicada à apresentação da teoria geral de sincronização de dois osciladores de fase, aplicando os resultados para relógios de pêndulo e metrônomos. A última seção é devotada às nossas Conclusões.

\section{Relógios de Pêndulo e sua Sincronização}

Christiaan Huygens (1629-1695) foi um dos nomes mais destacados da Física, Astronomia e Matemática do Século XVII [7. Uma das principais contribuições de Huygens foi a invenção do relógio de pêndulo em 1656 [Fig. 1]. Pouco tempo depois, o artesão holandês Salomon Coster construiu um relógio de pêndulo baseado no projeto de Huygens, e que atrasava apenas oito minutos por semana, uma precisão fantástica para sua época. Em 1673 Huygens publicou uma de suas obras mais importantes

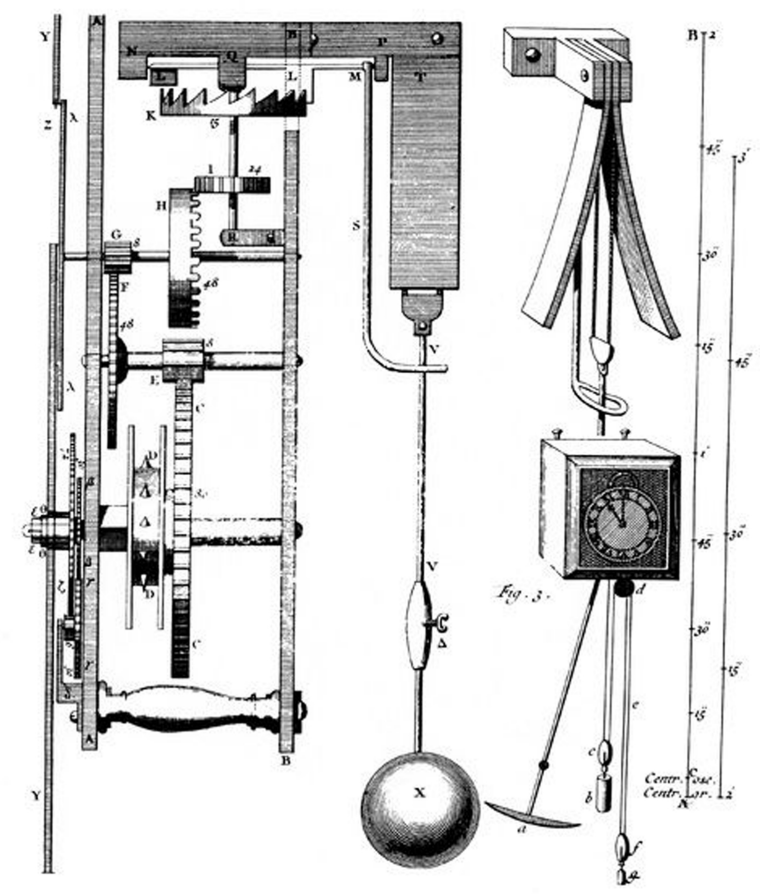

Figura 1: Relógio de pêndulo inventado por Huygens. Ilustração extraida da obra Horologium Oscillatorium [8].

(Horologium Oscillatorium), contendo suas investigações sobre os relógios de pêndulo [8,9]. Vale a pena mencionar que, juntamente com o "Discurso sobre as Duas Novas Ciências" de Galileo (1638) e o Principia Mathematica de Newton (1687), o livro de Huygens é considerado uma das obras fundamentais da Mecânica Clássica [10].

A necessidade de construir relógios os mais precisos possíveis era determinada basicamente pelas necessidades da navegação. A determinação da longitude era feita, na era clássica da navegação, pela comparação entre o meio-dia local e a hora em relação ao meridiano de Greenwich [11]. Huygens continuou aperfeiçoando seu invento tendo em vista o uso de relógios de pêndulo em alto mar.

A história do relógio de pêndulo começa, na verdade, em 1583, quando o então jovem Galileo Galilei postulou que o período de um pêndulo é independente da sua amplitude, desde que essa seja suficientemente pequena (isocronismo do pêndulo). Nessa aproximação o período das oscilações é dado por 12

$$
T=2 \pi \sqrt{\frac{\ell}{g}}
$$

onde $\ell$ é o comprimento do pêndulo e $g$ a aceleração da gravidade. Para $\ell \approx 1 \mathrm{~m}$ o período é igual a $2 \mathrm{~s}$. No entanto, devido aos inevitáveis atritos (com o ar, no ponto de suspensão, etc.) é necessário um aporte externo de energia para manter as oscilações do pêndulo por mais tempo. 
Esse aporte de energia, no relógio de pêndulo de Huygens, é fornecido pela lenta queda de um peso. Além disso, a queda é limitada por um mecanismo de escape do tipo âncora-roda dentada. A âncora é fixada na haste do pêndulo e oscila junto com ele [Fig. 2]. A roda dentada é ligada a uma polia, na qual um fio vai se desenrolando à medida em que o peso cai lentamente.

O movimento de rotação da roda dentada é interrompido pelo seu engajamento com a âncora, o que faz com que a haste do pêndulo oscile sempre com a mesma amplitude, compensando a perda de energia devido aos vários tipos de atrito existentes no sistema. Supondo um período para o pêndulo de $T=2 \mathrm{~s}$, como a âncora se engaja com a roda dentada duas vezes por ciclo de oscilação, o engajamento se dá uma vez por segundo, gerando assim o "tique" do relógio ( o "taque" é o outro engajamento). Além disso, o movimento da roda dentada é transmitido ao ponteiro dos segundos do relógio, que gira 1/60 de um círculo em cada "tique" do pêndulo.

Outra importante contribuição dada por Huygens ao desenvolvimento do relógio de pêndulo foi sua investigação sobre o problema da tautócrona. Uma partícula que desce por uma curva na forma de uma ciclóide, sob a ação do campo gravitacional uniforme, chega à parte mais baixa da curva num intervalo de tempo que não depende da posição inicial. Modificando o movimento do

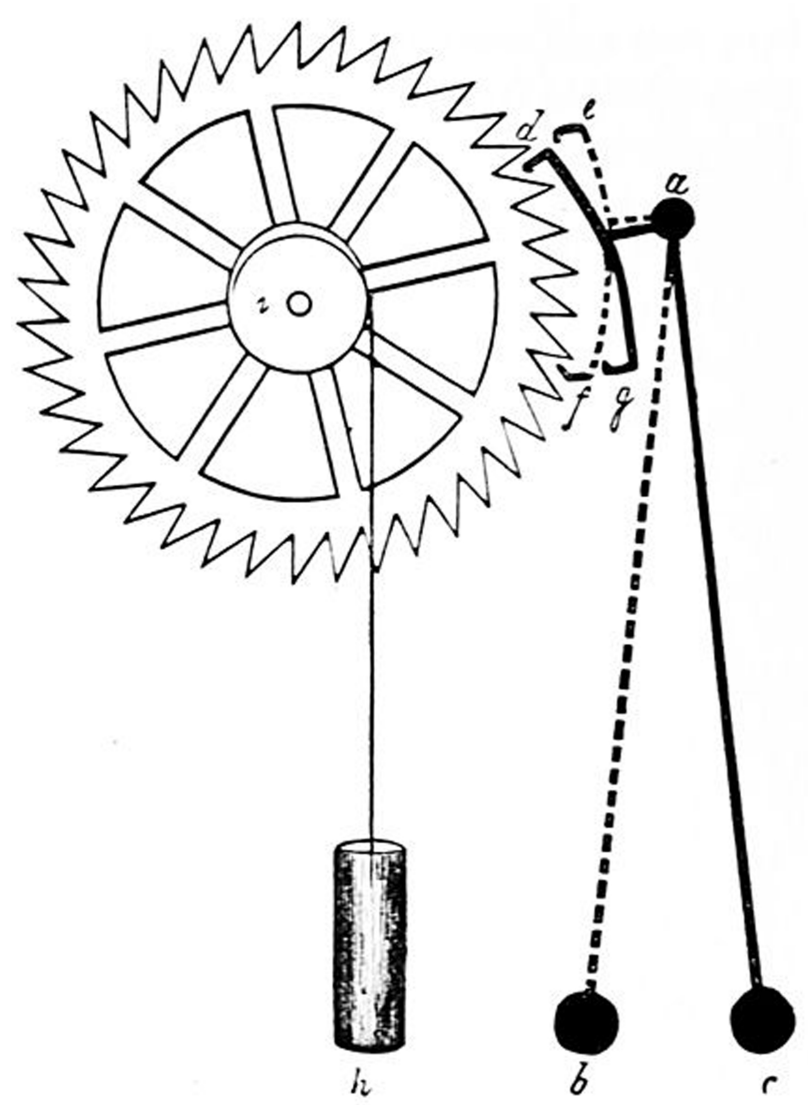

Figura 2: Mecanismo de escape do tipo âncora no relógio de pêndulo. Ilustração extraída da Ref. [13]. pêndulo para esse objetivo, este passa a ser isócrono para amplitudes maiores [7].

O próprio Huygens observou a sincronização de dois relógios de pêndulo presos a um suporte comum. Numa carta a seu pai, escrita em 1665 (há uma tradução dessa carta para o inglês no Apêndice A da referência [1] ), Huygens conta que, enquanto estava doente de cama, ficou observando o comportamento de dois relógios de pêndulo suspensos na parede do seu quarto e separados por uma distância de cerca de meio metro. Mesmo que, inicialmente, os pêndulos oscilassem a diferentes frequências, com o passar do tempo eles sincronizavam, fenômeno a que Huygens se referiu como "simpatia dos relógios" (sympathie des horloges).

Huygens posteriormente suspendeu os relógios de pêndulo por meio de um suporte comum [Fig. 3], observando os mesmos resultados. Na sua obra Horologium Oscillatorium, publicada em 1673 [8], Huygens descreve detalhadamente as suas observações. Os dois pêndulos suspensos por uma tábua sincronizavam suas frequências pois seus "tique-taques" eram ouvidos simultaneamente. Além disso, os pêndulos passavam a oscilar em anti-fase: quando um deles estava na sua posição máxima à esquerda o outro ficava à direita, e vice-versa [Fig. 4(b)].

A sincronização dos relógios de pêndulo era vista por Huygens não só como um fenômeno interessante, mas

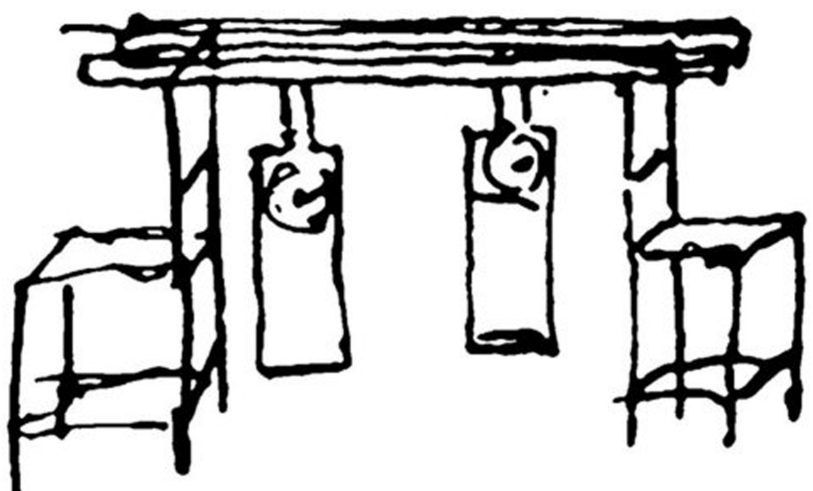

Figura 3: Desenho original de Christiaan Huygens mostrando dois relógios de pêndulo presos a um suporte comum. Reproduzido de [9].
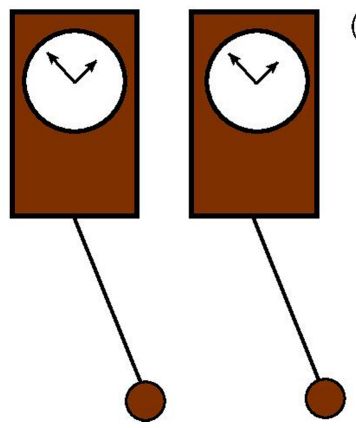

(a)
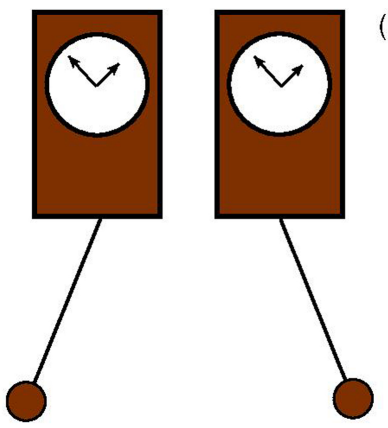

(b)

Figura 4: Figura esquemática de dois relógios de pêndulo oscilando sincronizadamente (a) em fase (b) em anti-fase. 
também como uma qualidade de redundância. Usando dois pêndulos acoplados como relógios marítimos, se um deles parasse ou precisasse de alguma manutenção, o outro poderia continuar a ser usado para a determinação da longitude.

Huygens também deu uma explicação para esses fenômenos, sugerindo que eles são causados por uma interação mecânica entre os pêndulos, transmitida pelo suporte comum. De fato, a sincronização entre os pêndulos é um fenômeno causado pelo movimento às vezes quase imperceptível do suporte devido aos impulsos mecânicos transmitidos a ele pelos pêndulos. Na linguagem moderna, dizemos que os pêndulos estão fracamente acoplados pelo suporte.

As observações de Huygens foram reproduzidas experimentalmente por Bennett e colaboradores, que usaram dois relógios de pêndulo idênticos suspensos por um a barra montada sobre rolamentos [14]. Um dos resultados mais interessantes é que a sincronização em anti-fase observada por Huygens só é possível se a diferença entre as frequências dos pêndulos for da ordem de $0,07 \%$, ou seja, se os relógios forem praticamente idênticos.

Um trabalho recente estudou quantitativamente o acoplamento de dois relógios de pêndulo suspensos por um suporte de massa finita 15. Conforme a razão entre as massas do pêndulo e do suporte, podem ser observadas tanto a sincronização em-fase como em anti-fase - Quando a massa do suporte é relativamente grande ou pequena observa-se sincronização em-fase [Fig. 4(a)] ou anti-fase [Fig. 4(b)], respectivamente. Uma revisão abrangente das pesquisas teóricas e experimentais sobre sincronização de relógios de pêndulo pode ser encontrada na Referência 4.

\section{Descrição Matemática do relógio de pêndulo}

O elemento fundamental de um relógio de pêndulo é o chamado pêndulo físico ou composto [16. Para os nossos propósitos neste trabalho, será suficiente introduzirmos ideias básicas da dinâmica não-linear, como a de ciclo-limite, para entender de forma qualitativa o comportamento do relógio de pêndulo como um oscilador de fase. Maiores detalhes sobre a descrição de sistemas com ciclos-limite podem ser encontrados na Referência [17].

\subsection{Pêndulo físico}

Um pêndulo físico consiste de uma haste rígida com uma massa fixada em sua extremidade. O momento de inércia será $I=m \ell^{2}$, onde $m$ é a massa do sistema e $\ell$ o raio de giração, aproximadamente igual ao comprimento da haste, se esta for suficientemente leve [vide Ref. [12], pg. 89]

A posição da haste em relação à vertical será descrita pelo ângulo $\phi$, onde $-\pi<\phi \leq \pi$. Considerando o torque da força gravitacional, a equação de movimento para o pêndulo é

$$
\frac{d^{2} \phi}{d t^{2}}=-\omega_{0}^{2} \sin \phi
$$

onde a frequência (angular) natural é

$$
\omega_{0}=\frac{2 \pi}{T}=\sqrt{\frac{g}{\ell}} .
$$

No caso de pequenas oscilações (tipicamente $\phi<5^{\circ}$ para relógios de pêndulo) fazemos a aproximação $\sin \phi \approx$ $\phi$, de modo que 22 reduz-se à equação de um oscilador harmônico, cuja solução é

$$
\begin{aligned}
& \phi(t)=\Phi \cos \left(\omega_{0} t+\psi\right), \\
& \dot{\phi}(t)=-\omega_{0} \Phi \sin \left(\omega_{0} t+\psi\right),
\end{aligned}
$$

onde $\Phi$ e $\psi$ são constantes, determinadas pelas condições iniciais $(\phi(0), \dot{\phi}(0))$.

Quadrando as equações (4) e (5) e somando os resultados membro-a-membro obtemos

$$
\frac{\phi^{2}}{\Phi^{2}}+\frac{(\dot{\phi})^{2}}{\omega_{0}^{2} \Phi^{2}}=1,
$$

que é a equação de uma elipse no plano de fase $\phi$ versus $\dot{\phi}$, com centro na origem e semieixos $\Phi$ e $\omega_{0} \Phi$ [Fig. [5(a)]. À cada condição inicial $(\phi(0), \dot{\phi}(0))$ corresponde uma trajetória diferente, associada a um dado valor da energia do sistema.

\subsection{Pêndulo amortecido}

Uma das principais causas do amortecimento do pêndulo é a resistência do ar ao seu movimento. Podemos supor que essa força (e o consequente torque) seja proporcional à velocidade angular, de modo que a equação de movimento do pêndulo amortecido será (no limite de pequenas oscilações)

$$
\frac{d^{2} \phi}{d t^{2}} \approx-b \frac{d \phi}{d t}-\omega_{0}^{2} \phi
$$

onde $b>0$ é o coeficiente de amortecimento viscoso.

O estudo completo do movimento harmônico amortecido contempla várias situações. No entanto, se o amortecimento for relativamente pequeno $\left(b \ll 2 \omega_{0}\right)$, a solução para o oscilador (sub-amortecido) será [vide [16], pg. 68]

$$
\phi(t)=\Phi e^{-b t / 2} \cos (\omega t+\psi),
$$
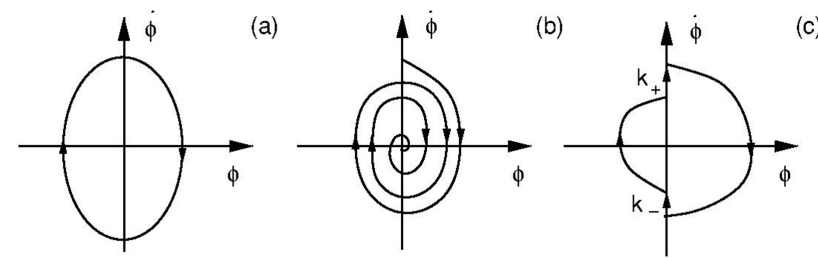

(c)

Figura 5: Plano de fase de um pêndulo nos casos (a) nãoamortecido; (b) amortecido; (c) forçado externamente por um mecanismo de escape (do tipo âncora-roda dentada). 
onde

$$
\omega=\sqrt{\omega_{0}^{2}-\frac{b^{2}}{4}} \approx \omega_{0}\left(1-\frac{b^{2}}{8 \omega_{0}^{2}}\right),
$$

usando a aproximação binomial.

A solução (8) representa uma oscilação harmônica com uma amplitude que cai exponencialmente com o tempo, e cuja frequência é ligeiramente menor do que $\omega_{0}$. Como tanto a posição como a velocidade angular tendem a zero quando $t \rightarrow \infty$, a trajetória no plano de fase deixa de ser uma curva fechada e passa a ser uma espiral que converge assintoticamente para o origem [Fig. 5(b)].

\subsection{Pêndulo amortecido com mecanismo de escape}

Para compensar a perda de energia devido ao amortecimento e manter uma oscilação estacionária, é necessário um aporte externo de energia ao pêndulo. No relógio de pêndulo de Huygens esse aporte é produzido pela lenta queda de um peso, que é transmitida ao pêndulo por um mecanismo de escape. Um dos destes mecanismos baseia-se no sistema âncora-roda dentada: cada vez que um dente da roda se engaja com a âncora um pequeno impulso angular $p(\phi, \dot{\phi})$ é transmitido ao pêndulo.

Pode-se configurar o mecanismo de escape de modo que este atue duas vezes por ciclo de oscilação de modo que um impulso angular $k_{+}$seja fornecido quando $\phi=0$ e $\dot{\phi}>0$, e outro impulso $k_{-}$quando $\phi=0$ e $\dot{\phi}<0$. O impulso $k_{+}$provoca um aumento súbito na velocidade angular, ao passo que $k_{-}$uma diminuição da mesma. O escape do tipo âncora, de fato, produz não só um avanço do ponteiro do segundo, mas também um pequeno recuo. Esse problema pode ser contornado usando um mecanismo diferente de escape chamado "pulso morto" (dead beat), e que consiste num único impulso aplicado [4].

A equação de movimento do pêndulo amortecido com escape do tipo âncora pode ser escrita como 18

$$
\ddot{\phi}+b \dot{\phi}+\omega_{0}^{2} \phi \approx \frac{1}{\Delta t} p(\phi, \dot{\phi}),
$$

onde $\Delta t$ é o intervalo de tempo ao longo do qual ambos os impulsos angulares são aplicados ao pêndulo, e

$$
p(\phi, \dot{\phi})= \begin{cases}k_{+} \delta(\phi), & \text { se } \dot{\phi}>0, \\ k_{-} \delta(\phi), & \text { se } \dot{\phi}<0,\end{cases}
$$

onde, sendo $n=1,2, \ldots$,

$$
\delta(\phi)= \begin{cases}1, & \text { se }|t-(2 \pi n / \omega)|<(\Delta t) / 2, \\ 0, & \text { caso contrário. }\end{cases}
$$

O efeito destes impulsos angulares sobre o movimento do pêndulo pode ser apreciado no plano de fase: a trajetória tem duas partes suaves, que são segmentos de espirais. Estas partes são conectadas por dois trechos retilíneos correspondendo aos impulsos $k_{+}$e $k_{-}$aplicados quando $\phi=0$ [Fig. 5.c)]. Vamos supor que o escape seja projetado de tal modo que $k_{+}>k_{-}$.

\subsection{Ciclos-limite e fases}

A curva fechada na Fig. 5(c) é uma solução estacionária da equação (10) chamada ciclo-limite. Os ciclos-limite são curvas fechadas correspondentes a órbitas periódicas. Além disso os ciclos-limite são curvas isoladas, ao contrário das elipses do caso sem atrito [Fig. 5(a)]. Enquanto estas últimas são curvas fechadas com outras similares infinitamente próximas a elas (cada qual com diferentes valores da energia), os ciclos-limite são isolados, não havendo outras soluções similares em sua vizinhança imediata 17 .

Em geral, oscilações auto-sustentadas, ou seja, com um aporte externo de energia que compensa as perdas devido a amortecimentos variados, são descritas no plano de fase por ciclos-limite. É necessário, ainda, que este ciclo-limite seja estável, ou seja, que condições iniciais na vizinhança do ciclo-limite produzam trajetórias que convergem assintoticamente a ele. O ciclo-limite do pêndulo descrito pela Eq. (10) é estável, o que foi mostrado por Denny [18] e por Llibre e Teixeira [19], usando diferentes métodos da Dinâmica Não-Linear.

É importante destacar que a descrição do movimento do relógio do pêndulo como um ciclo-limite equivale a uma redução dimensional. A equação de movimento 10 é um sistema bidimensional, enquanto o movimento sobre o ciclo-limite é unidimensional. Essa redução é possível porque o sistema é dissipativo, ou seja, a energia não é conservada.

\section{Metrônomos e sua Sincronização}

O metrônomo é um dispositivo que emite um som ("clique") a intervalos regulares e ajustáveis de tempo, cuja pulsação é usualmente medida em batimentos por minuto (bpm). Os metrônomos são muito usados no aprendizado de instrumentos musicais, dentre outras aplicações. Embora sejam muito mais comuns na atualidade os metrônomos eletrônicos e digitais, vamos considerar neste trabalho os metrônomos mecânicos, que consistem essencialmente numa haste com um peso acima do centro de massa, cuja posição ao longo da haste pode ser ajustada para controlar a pulsação. Se o peso é deslizado em direção à extremidade livre da haste, a pulsação é diminuida, enquanto se for deslizada na direção oposta, a pulsação aumenta.

Essa configuração é conhecida também como pêndulo invertido. Na verdade, além do peso deslizável, há também um segundo peso fixo na outra extremidade da haste, e situado no interior do metrônomo. As oscilações do pêndulo invertido são mantidas por meio de um mecanismo de escape baseado no distensionamento de uma mola espiral. Os cliques do metrônomo equivalem, assim, aos tique-taques do relógio do pêndulo.

Caríssimos no passado, quase um ítem de colecionador, atualmente ótimos metrônomos mecânicos estão disponíveis no mercado por preços relativamente baixos (a 
partir de $\mathrm{R} \S 300,00$ ), o que facilita o seu uso didático. A pulsação nestes metrônomos vai desde 40 bpm (largo) até $208 \mathrm{bpm}$ (prestissimo).

Há dois tipos básicos de acoplamento entre metrônomos que têm sido considerados. Se os metrônomos estiverem colocados numa base que pode deslizar com pouco atrito sobre uma superfície horizontal, observa-se que os metrônomos com frequências praticamente idênticas sincronizam em-fase [Fig. 6(a)]. Já se os metrônomos estiverem numa base com bastante atrito, limitando sua mobilidade, eles podem sincronizar anti-fase [Fig. $6(\mathrm{~b})]$ [20].

Assim como o relógio de pêndulo, as oscilações de um metrônomo com um mecanismo de escape e uma fonte de energia baseada numa mola espiral podem ser associadas a um ciclo-limite estável de um sistema dinâmico. Pantaleone modelou matematicamente as oscilações de um metrônomo por meio de uma equação de movimento que, após um certo número de aproximações e definições de variável, tem a forma da equação de van der Pol [21]

$$
\ddot{\phi}=-\mu\left(\phi^{2}-1\right) \dot{\phi}-\phi,
$$

onde $\phi$ é o ângulo entre a haste e a vertical, e $\mu$ é um parâmetro que contém informações sobre o mecanismo de escape assim como o amortecimento do pêndulo invertido.

A equação de van der Pol admite soluções estacionárias na forma de ciclos-limite estáveis, um exemplo sendo

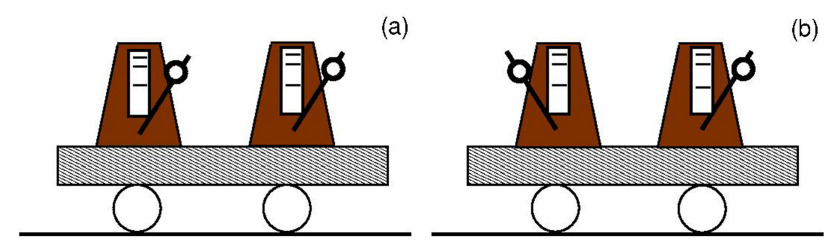

Figura 6: Sincronização de dois metrônomos acoplados por um suporte móvel: (a) em fase; (b) em anti-fase.

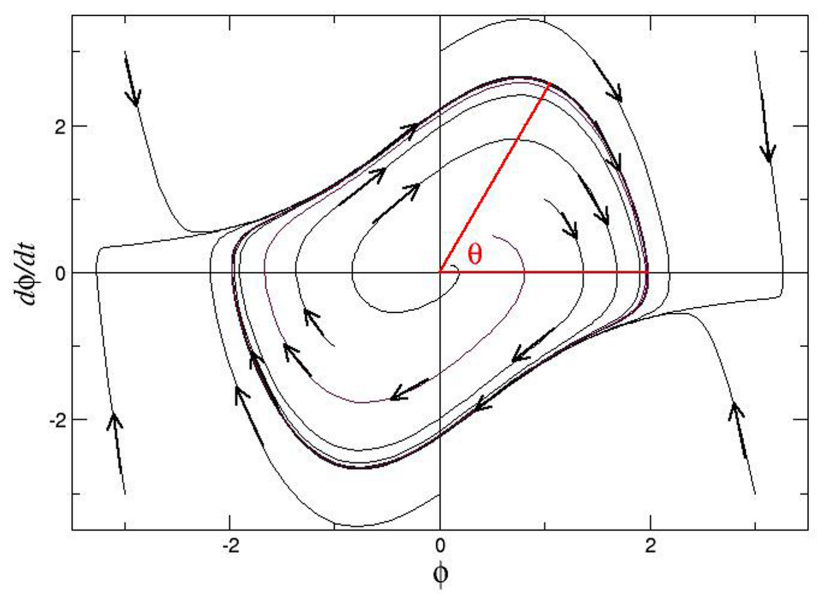

Figura 7: Ciclo-limite estável da equação de van der Pol (13) para $\mu=1$. São mostradas, também, alguma soluções que convergem assintoticamente para o ciclo-limite, bem como a definição da fase geométrica. mostrado na Fig. 7, que corresponde a uma solução numérica da equação (13) para o caso $\mu=1$, que corresponde ainda a uma oscilação do tipo pendular. Para valores mais altos do parâmetro $\mu$ a equação 13 pode também descrever osciladores de relaxação [22]. Um estudo mais detalhado da equação de Van der Pol e de suas aplicações, enfatizando oscilações de relaxação, pode ser encontrado na Referência 21].

Assim, o que os relógios de pêndulo e os metrônomos têm em comum é o fato de ambos serem osciladores auto-excitáveis, cujo movimento no plano de fase é um ciclo-limite estável. O movimento sobre o ciclo-limite, supondo que este seja deformável a um círculo, pode ser descrito por meio de uma fase geométrica

$$
\tan \theta(t)=\frac{\dot{\phi}(t)-c_{1}}{\phi(t)-c_{2}},
$$

onde $\left(c_{1}, c_{2}\right)$ são coordenadas de um ponto no interior do ciclo. Na Fig. 7 mostramos essa definição, tomando $c_{1}=c_{2}=0$. Em situações onde a fase geométrica não pode ser definida de maneira unívoca por (14), outras definições de fase podem ser empregadas [1].

De acordo com a definição (14), a fase aumenta em $2 \pi$ radianos na medida em que o ciclo-limite seja percorido. Para um oscilador uniforme, onde a frequência (angular) $\omega$ seja constante, a equação de evolução da fase é

$$
\frac{d \phi}{d t}=\omega
$$

O acoplamento entre os metrônomos no trabalho de Pantaleone foi modelado por meio do movimento do suporte móvel sobre os quais eles estão apoiados [20]. Foi observada experimentalmente a sincronização em fase dos metrônomos [Fig. 6(a)] na maioria dos casos. Em algumas condições particulares (por exemplo, aumentando o amortecimento no rolamento do suporte) foi também observada uma sincronização do tipo anti-fase [Fig. 6(b)].

\section{Sincronização de Osciladores de Fase}

\subsection{Tratamento geral}

Um dos aspectos comuns ao relógio de pêndulo e o metrônomo é a presença de oscilações autosustentadas com uma frequência bem-definida. O movimento de ambos os osciladores, no plano de fase, está restrito a ciclos-limite estáveis. Dois osciladores desse tipo podem ser descritos por duas fases $\theta_{1}$ e $\theta_{2}$. Por conveniência supomos que $-\pi<\theta_{1,2} \leq \pi$. Se as frequências associadas a cada um deles forem $\omega_{1}$ e $\omega_{2}$ (supostas constantes) as equações para os osciladores desacoplados são

$$
\frac{d \theta_{1}}{d t}=\omega_{1}, \quad \frac{d \theta_{2}}{d t}=\omega_{2}
$$

O acoplamento entre osciladores mecânicos é devido à propagação de impulsos ao longo do suporte no qual eles são colocados ou fixados. Como estes impulsos dependem 
das fases dos osciladores, podemos escrever as equações para os osciladores acoplados na seguinte forma geral [1]

$$
\begin{aligned}
& \frac{d \theta_{1}}{d t}=\omega_{1}+\varepsilon A\left(\theta_{1}, \theta_{2}\right), \\
& \frac{d \theta_{2}}{d t}=\omega_{2}+\varepsilon B\left(\theta_{2}, \theta_{1}\right),
\end{aligned}
$$

onde $A$ e $B$ são funções períodicas em $\theta_{1,2}$, com período $2 \pi$.

A intensidade do acoplamento entre os osciladores é regulada pelo parâmetro $\varepsilon$, que pode ter valores positivos ou negativos, mas cujo módulo é sempre pequeno $(|\varepsilon| \ll$ 1) (interação "fraca"). Uma ressonância generalizada entre os osciladores ocorre sempre que as frequências sejam comensuráveis, ou seja, que existam inteiros primos entre si $n$ e $m$ tais que

$$
n \omega_{1}-m \omega_{2}=0
$$

Se as frequências estiverem próximas à ressonância $\left(n \omega_{1}-m \omega_{2} \approx 0\right)$ os termos ressonantes nas séries (39)(40) oscilam lentamente com o tempo, enquanto os nãoressonantes variam rapidamente e anulam-se quando tomamos uma média no tempo. Após esse procedimento obtemos as seguintes equações médias para os osciladores de fase (vide Apêndice para detalhes do cálculo)

$$
\begin{aligned}
\frac{d \theta_{1}}{d t} & =\omega_{1}+\varepsilon g_{1}(\varphi), \\
\frac{d \theta_{2}}{d t} & =\omega_{2}+\varepsilon g_{2}(-\varphi),
\end{aligned}
$$

onde definimos a diferença de fase generalizada

$$
\varphi=n \theta_{1}-m \theta_{2},
$$

e os termos $g_{1,2}$ são médias temporais de $A$ e $B$, dados pelas equações (45)-(46) do Apêndice.

Multiplicando (20) por $n$, 21 por $m$ e subtraindo membro-a-membro os resultados obtemos, em vista de (22), a equação que governa a evolução da diferença de fase

$$
\frac{d \varphi}{d t}=-\Delta \omega+\varepsilon g(\varphi),
$$

onde definimos o descasamento generalizado de frequências

$$
\Delta \omega=m \omega_{2}-n \omega_{1},
$$

assim como a função de acoplamento

$$
g(\varphi)=n g_{1}(\varphi)-m g_{2}(-\varphi)
$$

Vamos considerar o caso de ressonância simples $\left(\omega_{1}=\right.$ $\left.\omega_{2}\right)$ por ser o mais importante nas aplicações. Fazendo $n=m=1$ em 22, 24 e 25 temos

$$
\begin{aligned}
\varphi & =\theta_{1}-\theta_{2}, \\
\Delta \omega & =\omega_{2}-\omega_{1}, \\
g(\varphi) & =g_{1}(\varphi)-g_{2}(-\varphi),
\end{aligned}
$$

nas proximidades da ressonância exata.

Considerando que os osciladores sejam praticamente idênticos é razoável supor que o acoplamento seja simétrico: $g_{1}(\varphi)=g_{2}(\varphi)$, de modo que (28) implica em $g(\varphi)=-g(-\varphi)$. Uma função periódica simples com essa propriedade é: $g(\varphi)=\sin \varphi$, de modo que 23 fica

$$
\frac{d \varphi}{d t}=-\Delta \omega+\varepsilon \sin \varphi
$$

que é conhecida como equação de Adler 6 .

Devido ao acoplamento, as frequências dos osciladores são modificadas. De 20)-21 elas passam a ser dadas por

$$
\Omega_{1,2} \equiv\left\langle\dot{\theta}_{1,2}\right\rangle=\omega_{1,2}+\frac{\varepsilon}{2} \sin ( \pm \varphi)
$$

onde $\langle\ldots\rangle$ é uma média temporal, dada por 42. A diferença entre as frequências não é mais exatamente igual a zero, mas passa a depender da diferença de fase:

$$
\Omega_{2}-\Omega_{1}=\varepsilon \sin \varphi
$$

\subsection{Sincronização de fases e frequências}

As propriedades da equação de Adler foram discutidas num trabalho anterior, no contexto da influência de um forçamento externo sobre um oscilador de fase [6]. Sem perda de generalidade podemos supor que $\Delta \omega>0$. Assim, dois casos distintos apresentam-se:

\subsubsection{Interação atrativa $(\varepsilon<0)$}

Para analisar a evolução temporal de $\varphi$ nós podemos empregar o diagrama de fase da Fig. 8(a), que é o gráfico do segundo membro da equação de Adler (29). O sinal de $\dot{\varphi}$ indica o sentido do fluxo $\varphi(t)$. Os pontos fixos da equação de Adler, denotados por $\varphi^{*}$, satisfazem a relação

$$
\sin \varphi^{*}=\frac{\Delta \omega}{\varepsilon} .
$$

Uma vez que $\left|\sin \varphi^{*}\right| \leq 1$ obtemos a seguinte condição para a existência de pontos fixos

$$
\Delta \omega \leq|\varepsilon|
$$

Se (33) for satisfeita, há duas soluções para (32): $\varphi_{1,2}^{*}$. Pela inspeção do diagrama de fase [Fig. 8(a)] resulta que $\varphi_{1}^{*}$ é um ponto fixo estável, ou seja, quase todas as condições iniciais $\varphi(t=0)$ aproximam-se de $\varphi_{1}^{*}$ quando $t \rightarrow \infty$. A exceção é justamente o segundo ponto fixo $\varphi_{2}^{*}$, que é instável. Para $\varepsilon<0$ o ponto fixo estável é tal que $-\pi / 2<\varphi_{1}^{*}<\pi / 2$, de modo que a diferença de fase

$$
\theta_{1}-\theta_{2}=\varphi_{1}^{*}
$$

permanece constante com o passar do tempo, o que caracteriza o chamado travamento de fase (phase locking).

Se $\Delta \omega=0$ (ressonância exata), de (32) temos que $\varphi_{1}^{*}=0$, ou seja $\theta_{1}=\theta_{2}$. Nesse caso as fases tornam-se 

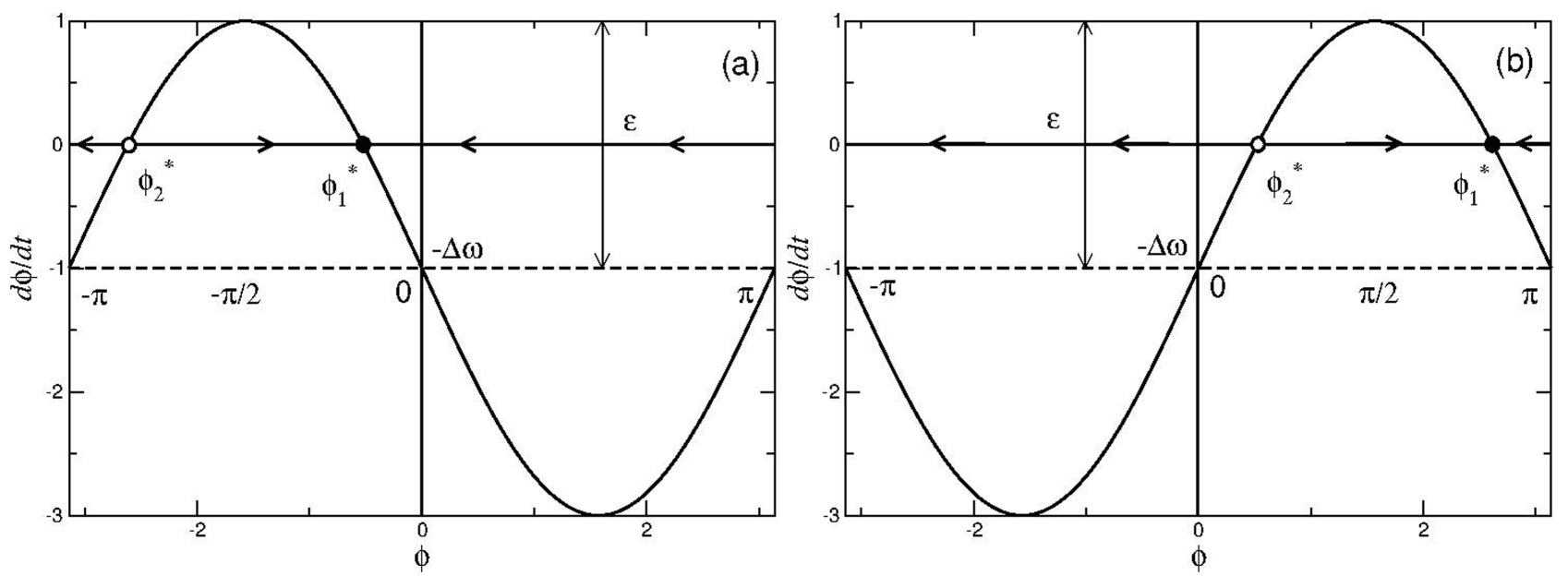

Figura 8: Diagramas de fase para a equação de Adler nos casos de interação (a) atrativa $(\varepsilon<0)$; (b) repulsiva $(\varepsilon>0)$.

iguais, e podemos pensar que elas se atraem mutuamente devido ao acoplamento (daí termos chamado esse caso de interação atrativa). Temos, então, uma sincronização em-fase dos osciladores. Esse é o caso ilustrado pelos relógios de pêndulo [Fig. 4(a)] e metrônomos [Fig. 6(a)].

Usando (30), 27) e (32) as frequências perturbadas são iguais:

$$
\Omega_{1}=\Omega_{2}=\frac{\omega_{1}+\omega_{2}}{2},
$$

tal que $\omega_{1}<\Omega_{1,2}<\omega_{2}$. Assim, a sincronização de fases implica na sincronização de frequências para os osciladores.

\subsubsection{Interação repulsiva $(\varepsilon>0)$}

O diagrama de fase correspondente a esse caso está ilustrado na Fig. 8(b). Aqui, também, haverá um ponto fixo estável $\left(\varphi_{1}^{*}\right)$ e outro instável $\left(\varphi_{2}^{*}\right)$, tal que $\pi / 2<\varphi_{1}^{*}<$ $3 \pi / 2$. Nesse caso a diferença de fase continua constante (travamento de fase). No entanto, quando a condição de ressonância $\Delta \omega=0$ é satisfeita, teremos agora que $\varphi_{1}^{*}=\pi$, tal que

$$
\theta_{1}=\pi+\theta_{2},
$$

de sorte que os osciladores estão sincronizados em antifase, ou seja, eles repelem-se mutuamente devido ao acoplamento. Esse tipo de interação repulsiva é obsevado tanto em relógios de pêndulo [Fig. 4(b)] como metrônomos [Fig. 6(b)]. O comportamento das frequências perturbadas dos osciladores é similar ao caso de interação atrativa.

Finalmente, vamos considerar o caso em que a condição (33) não é satisfeita, ou seja, quando a diferença entre as frequências for relativamente grande, ou seja, se $\Delta \omega>|\varepsilon|$. Não havendo ponto fixo na equação de Adler não haverá travamento de fases, ou seja, a diferença de fase entre os osciladores poderá aumentar ou diminuir com o passar do tempo.

Caso $\Delta \omega$ seja apenas ligeiramente superior a $|\varepsilon|$ formase um "gargalo" no diagrama de fase, próximo ao que seria o ponto fixo $\sin \varphi_{1,2}^{*}= \pm 1$. Assim, quando a diferença de fases estiver próxima a esse "fantasma" do ponto fixo haverá um travamento temporário entre as fases. Logo que o gargalo tenha sido ultrapassado, no entanto, a diferença de fases voltará a variar com o tempo. Esse tipo de situação caracteriza os chamados deslizamentos de fase (phase slips) [1].

\section{Conclusões}

Relógios de pêndulo e metrônomos são osciladores autoexcitados e podem ser descritos matematicamente por uma fase que parametriza o movimento ao longo de um ciclo-limite. Dois osciladores acoplados podem ser, assim, modelados de forma bastante geral usando equações médias próximas a uma ressonância generalizada (relação comensurável entre as frequências não-perturbadas).

No caso de estarmos próximos a uma ressonância simples, as equações médias mostram que as fases dos osciladores são travadas pelo acoplamento, ou seja, a diferença de fase torna-se constante. Além disso, as frequências perturbadas dos osciladores tornam-se iguais à média das frequências não-perturbadas. Temos, portanto, sincronização de frequências e travamento de fases.

Há uma diferença fundamental no comportamento dos osciladores em relação ao tipo de acoplamento, caracterizado pelo sinal da intensidade do acoplamento $\varepsilon$. Para interações atrativas $(\varepsilon<0)$ as fases dos osciladores tendem a se igualar (sincronização em-fase), ao passo que para interações repulsivas $(\varepsilon>0)$ as fases tendem a se afastar (sincronização anti-fase). Ambos os casos são observados no comportamento de relógios de pêndulo e metrônomos acoplados. Nossa análise quantitativa está de acordo com os resultados quantitativos de Pena-Ramirez e colaboradores, que associam a sincronização em-fase com a diminuição da intensidade de acoplamento e a sincronização anti-fase com o aumento na intensidade de acoplamento [15]. 
Empregando equações médias nós levamos em conta apenas os termos próximos às ressonâncias, já que os termos não-ressonantes anulam-se quando as médias temporais são tomadas. No entanto, se os termos fora da ressonância forem levados em conta, o travamento de fase será válido apenas de maneira aproximada.

Para osciladores pendulares, como relógios de pêndulo e metrônomos (que são pêndulos invertidos), as funções de acoplamento têm poucos harmônicos, e o efeito dos termos não-ressonantes é relativamente pequeno, podendo ser ignorado. Este é o caso abordado no presente trabalho.

Há, porém, osciladores de relaxação, como o vaso de tântalo e o monjolo, para os quais as funções de acoplamento têm uma infinidade de harmônicos (exemplos são funções do tipo dente-de-serra ou pulsos quadrados) 22. $\mathrm{Na}$ ressonância entre osciladores de relaxação acoplados, o travamento de fase poderá não existir, havendo flutuações da diferença de fase em torno dos valores previstos pelas equações médias.

\section{Agradecimentos}

$\mathrm{O}$ autor agradece ao $\mathrm{CNPq}$ pelo apoio financeiro para a realização deste trabalho (bolsa PQ1B, proc. 301019/2019$3)$.

\section{Apêndice: Obtenção das equações médias no caso ressonante}

Nas equações (17)-18 para dois osciladores de fase acoplados, os termos de acoplamento são periódicos nas duas fases e, portanto, podem ser expandidos em séries de Fourier 1]:

$$
\begin{aligned}
& A\left(\theta_{1}, \theta_{2}\right)=\sum_{k=-\infty}^{\infty} \sum_{\ell=-\infty}^{\infty} a_{k \ell} e^{i\left(k \theta_{1}+\ell \theta_{2}\right)} \\
& B\left(\theta_{2}, \theta_{1}\right)=\sum_{\ell=-\infty}^{\infty} \sum_{k=-\infty}^{\infty} b_{\ell k} e^{i\left(\ell \theta_{2}+k \theta_{1}\right)}
\end{aligned}
$$

onde $a_{k \ell}$ e $b_{k \ell}$ são coeficientes de Fourier, cujas expressões matemáticas dependem dos tipos dos osciladores e do seu acoplamento.

$\mathrm{Na}$ aproximação de acoplamento fraco entre os osciladores $(\varepsilon \ll 1)$ podemos, em primeira aproximação, considerar que as fases $\theta_{1,2}$ evoluem de acordo com as equações desacopladas 16 :

$$
\theta_{1}=\theta_{10}+\omega_{1} t, \quad \theta_{2}=\theta_{20}+\omega_{2} .
$$

Supondo, sem perda de generalidade, que $\theta_{10}=\theta_{20}=$ 0 , as expansões de Fourier (36)-37) ficam

$$
\begin{aligned}
& A\left(\theta_{1}, \theta_{2}\right)=\sum_{k, \ell} a_{k \ell} e^{i\left(k \omega_{1}+\ell \omega_{2}\right) t}, \\
& B\left(\theta_{2}, \theta_{1}\right)=\sum_{k, \ell} b_{\ell k} e^{i\left(\ell \omega_{2}+k \omega_{1}\right) t} .
\end{aligned}
$$

Quando as frequências dos osciladores são comensuráveis na forma $n \omega_{1}-m \omega_{2}=0$, podemos separar os termos ressonantes e não-ressonantes em (39):

$$
\begin{aligned}
A\left(\theta_{1}, \theta_{2}\right)= & \sum_{k=n j, \ell=-m j} a_{n j,-m j} e^{i j\left(n \omega_{1}-m \omega_{2}\right) t} \\
& +\sum_{k \neq n j} \sum_{\ell \neq-m j} a_{k \ell} e^{i\left(k \omega_{1}+\ell \omega_{2}\right) t} .
\end{aligned}
$$

Definimos uma média temporal em relação aos dois períodos

$$
\begin{aligned}
& \left\langle A\left(\theta_{1}, \theta_{2}\right)\right\rangle=\frac{\omega_{1}}{2 \pi} \int_{0}^{2 \pi / \omega_{1}} d t \times \\
& \frac{\omega_{2}}{2 \pi} \int_{0}^{2 \pi / \omega_{2}} d t^{\prime} A\left(\theta_{1}(t), \theta_{2}\left(t^{\prime}\right)\right),
\end{aligned}
$$

tal que os termos não-ressonantes em (41), e que oscilam rapidamente com o tempo, têm média igual a zero, o que nos deixa apenas com os termos ressonantes:

$$
\left\langle A\left(\theta_{1}, \theta_{2}\right)\right\rangle=\sum_{j} a_{n j,-m j} e^{i j\left(n \omega_{1}-m \omega_{2}\right) t} .
$$

Analogamente obtemos, para o termo 40, o valor médio

$$
\left\langle B\left(\theta_{2}, \theta_{1}\right)\right\rangle=\sum_{j} b_{-m j, n j} e^{i j\left(m \omega_{2}-n \omega_{1}\right) t} .
$$

Definindo a diferença de fase generalizada $\varphi=n \theta_{1}-$ $m \theta_{2}$ os termos médios (43)- 44 são escritos

$$
\begin{array}{r}
\left\langle A\left(\theta_{1}, \theta_{2}\right)\right\rangle=g_{1}(\varphi)=\sum_{j} a_{n j,-m j} e^{i j \varphi}, \\
\left\langle B\left(\theta_{2}, \theta_{1}\right)\right\rangle=g_{2}(-\varphi)=\sum_{j} b_{-m j, n j} e^{-i j \varphi}
\end{array}
$$

tal que as equações médias são

$$
\begin{aligned}
\frac{d \theta_{1}}{d t} & =\omega_{1}+\varepsilon g_{1}(\varphi) \\
\frac{d \theta_{2}}{d t} & =\omega_{2}+\varepsilon g_{2}(-\varphi)
\end{aligned}
$$

\section{Referências}

[1] A. Pikowsky, M. Rosenblum e J. Kurths, Synchronization: a Universal Concept in Nonlinear Sciences (Cambridge University Press, Cambridge, 2001).

[2] J. Rayleigh, The Theory of Sound (MacMillan and Co. New York, 1945).

[3] E.V. Appleton, Proc. Cambridge Phil. Soc. 21, 231 (1922).

[4] M. Kapitaniak, K. Czolczynski, P. Perlikowski, A. Stefanski e T. Kapitaniak, Physics Reports 517, 1 (2012).

[5] A. Winfree, The Geometry of Biological Time (SpringerVerlag, Berlim, 2001), $2^{\text {a }}$ ed.

[6] R.L. Viana e F.F. Carvalho, Rev. Bras. Ensino Fís. 39, 3306 (2017). 
[7] A.E. Bell, Christian Huygens and the Development of Science in the Seventeenth Century (Edward Arnold \& Co., London, 1950).

[8] C. Huygens, Horologium Oscillatorium: sive de motu pendulorum ad horologia aptato demonstrationes geometricae (F. Muguet, Paris, 1658).

[9] C. Huygens, Oeuvres complétes de Christiaan Huygens (M. Nijhoff, La Haye, 1932), v. 17.

[10] A.E. Bell, Nature 148, 245 (1941).

[11] J.G. Yoder, Unrolling Time: Christiaan Huygens and the Mathematization of Nature (Cambridge University Press, Cambridge, 2004).

[12] H.M. Nussenzveig, Curso de Física Básica (Edgard Blücher, Sâo Paulo, 1986), v. 2.

[13] The Popular Science Monthly, disponível em: https: //archive.org/details/popularsciencemo29newy/ page/4/mode/2up

[14] M. Bennett, M.F. Schatz, H. Rockwood e K. Wiesenfeld, Proc. R. Soc. London A 458, 563 (2002).

[15] J. Pena-Ramirez, R.H.B. Fey e H. Nijmeijer, Chaos 23, 033118 (2013).

[16] K.R. Symon, Mecânica (Ed. Campus, Rio de Janeiro, 1982), $3^{\mathrm{a}}$ ed.

[17] L.H.A. Monteiro, Sistemas Dinâmicos (Editora da Física, São Paulo, 2007), $2^{\text {a }}$ ed.

[18] M. Denny, European Journal of Physics 23, 449 (2002).

[19] J. Llibre e M.A. Teixeira, European Journal of Physics 31, 1249 (2010)

[20] J. Pantaleone, American Journal of Physics 70, 992 (2002).

[21] R.L. Viana, Rev. Bras. Ens. Fis. 33, 3305 (2011).

[22] R.L. Viana, Rev. Bras. Ens. Fis. 33, 3304 (2011). 